\title{
Di Dante, Pellico, Petito e Francesca da Rimini. Qualche divagazione
}

\section{Francesco Cotticelli}

Seconda Università di Napoli

franzraf@libero.it

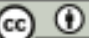

\section{Riassunto}

Riflettendo sulla parodia della Francesca da Rimini di Silvio Pellico (I8I5), cult play delle scene ottocentesche italiane, che Antonio Petito compose nel I866, il lavoro suggerisce di considerare l'attore-autore napoletano non solo una voce caustica nei confronti di tanto (vacuo) teatro del suo tempo, ma anche - per questa sottile vis polemica - un "interprete" inconsapevole ma lucido dello spirito militante dantesco.

Parole chiave: Petito; Francesca da Rimini; parodia; Napoli; Pellico; Teatro XIX secolo.

\begin{abstract}
Reflecting on the parody of Silvio Pellico's Francesca da Rimini (I815), cult play of the nineteenth Century Italian scenes, that Antonio Petito composed in I866, the work suggests to consider the neapoletan actor-author not just as a caustic voice towards so (inane) theatre of his time, but also - for this fine polemic vis - an unaware but polished "interpreter" of dante's militant spirit.
\end{abstract}

Keywords: Petito; Francesca da Rimini; parody; Naples; Pellico; XIX century Theatre. 
S arebbe fuorviante immaginare Petito fra gli appassionati esegeti di Dante $\checkmark$ nel cuore della stagione post-romantica italiana. Se un dialogo esiste con l'autore non è attraverso i suoi versi e il loro strepitoso revival alla luce della passione diffusa per le vicende del Medioevo, ma seguendo con acume e ironia l'uso drammaturgico (e non solo) che se ne realizzò sulle scene dell'intero paese. Uno straordinario serbatoio di perfette tragedie: trame e figure che rinviano alla Commedia arricchiscono una letteratura teatrale esile per quantità e costituzione, non di rado assurgendo a modelli per ulteriori episodi, e si prestano efficacemente alle alchimie poetiche e organizzative delle compagnie di tradizione. È il caso della Francesca da Rimini di Silvio Pellico, andata in scena per la prima volta a Milano il I8 agosto I8I5 e impostasi quindi rapidamente nel repertorio ${ }^{\mathrm{I}}$. Il celebre episodio del canto quinto dell'Inferno, con la rievocazione dell'amore infelice fra i due cognati affidato alla dolente voce della donna e intessuto di aforismi ed echi della produzione cortese, costituisce un ascendente di sicuro pregio e un "argomento" noto alle platee; Pellico, che ben altre scritture avrebbero immortalato fra i protagonisti del convulso Ottocento italiano, concentra risolutamente l'azione in pochi quadri e intorno a un gioco di figure essenziali, Lanciotto, il marito, Paolo e Francesca, appunto, e Guido, il padre di lei, dietro le quali non è difficile ravvisare le tipologie di ruoli che assicurano qualità e continuità allo spettacolo dei grandi attori. L'ammiccamento al sentimento nazionalistico nella tirata dell'eroe Paolo che rientra in patria (la "parlata sopra l'Italia»"), lo sfondo di guerre e contrasti passati e presenti su cui si staglia la sofferenza repressa di un microcosmo familiare, il linguaggio aulico e solenne in cui rifulgono stilemi danteschi accanto ai tragici coevi, l'impianto classicistico che si riflette nell'ossequio alle unità canoniche sono elementi di sicura presa sull'uditorio e garantiscono una funzionalità del testo al gusto declamatorio e a una recitazione dalle tinte forti, perfettamente assecondato dai primi interpreti, in particolare Luigi Domeniconi nei panni di Paolo e l'allor giovane Carlotta Marchionni. È un successo senza precedenti, e pressoché isolato anche nella carriera dello stesso autore, che si ripete grazie alla fascinazione che quelle parti esercitano su tutti i grandi attori ${ }^{3}$.

Significativo è il fatto che la tragedia sia riproposta al Teatro dei Fiorentini di Napoli il I3 luglio I860, grazie all'intraprendenza di uno dei più solerti impresari dell'epoca, Adamo Alberti, deciso a riportare nella capitale un ar-

I. Per Francesca da Rimini di Silvio Pellico si veda l'edizione Pellico (1985) da cui si cita. Si veda anche Borrelli (1995) nonché Farina (2006).

2. Così è definita la tirata di Paolo in Francesca da Rimini, atto I, scena quinta (Pellico 1985: 277).

3. Si veda Pellico (1985: 259-265). 
tista di grido come Tommaso Salvini affiancato da Fanny Sadowsky4, che si sarebbe stabilita permanentemente in città diventando a sua volta impresario prima del Teatro del Fondo, poi del Teatro Nuovo. Una fortunata serie di repliche e l'interesse sempre vivo per l'evento dimostrano l'intelligenza di una scelta non scontata, se si pensa che il dramma avrebbe potuto risentire di una certa vetustà a confronto con orientamenti decisamente più borghesi e che la memoria di recenti messinscene avrebbe potuto condizionare i favori del pubblico'. In un anno cruciale per i destini del Mezzogiorno, l'ennesima ripresa della Francesca da Rimini rilancia la natura di cult play, alimentando quasi un divismo a distanza che è buona parte del destino lusinghiero toccato alla tragedia. È il segno anche di una longevità ammirevole, tanto più che gli scenari esterni sono davvero mutati, e in maniera davvero radicale. Se nel I866 l'astro dei palcoscenici napoletani, Antonio Petito, decide di cimentarsi nella parodia dell'opera, i tempi sono maturi per consegnare anche il testo di Pellico alla teoria dei capolavori "stravisati" ad uso del teatro comico, proseguendo un ideale colloquio con autori e interpreti delle scene "ufficiali" italiani e stranieri'.

D'altronde, sin dalla fine del Settecento la parodia è assurta a genere privilegiato per l'ambito dialettale, forma distintiva di un mondo poeticoproduttivo che continua ad articolarsi intorno a una concezione prettamente attoriale del lavoro scenico, ispirata a una ridicolosità urbana, al tratteggio di maschere, al ricorso ancora cospicuo alle tecniche dell'improvvisazione (con ineludibili ricadute sulle effettive possibilità di ricostruzione di quei testi e di quelle esperienze di spettacolo). E la via "napoletana" al teatro, che sarebbe improprio tacciare di subalternità a canoni e a latitudini meglio accreditati, ed è piuttosto ricerca - o difesa - di un'alterità antica ${ }^{7}$. Il sistema della parodia vive - beninteso - della contiguità con il modello: qui si tratta di una duplice contiguità, se si considera la vicinanza nello spazio e nel tempo delle rappresentazioni "italiane" e la forte presenza di quel tema nell'immaginario, sia per la conoscenza di Dante sia per la familiarità con la rielaborazione di

4. Sulle circostanze delle riprese napoletane degli anni Sessanta cfr. Sapienza (2003: 45-59), cui si rinvia anche per un'analisi dettagliata della parodia petitiana. Per la Sadowsky si veda Seller (1989: 197-200).

5. Su questo problema, le memorie di Salvini e di Alberti, oltre ad Sapienza (2003), si veda Greco (1995: 137). Si tratta della nota al testo della Francesca da Rimini di Petito, riproposta alle pp. I27-I36: da questa edizione si cita. Al volume si rinvia tra l'altro per una lettura complessiva del fenomeno teatrale a Napoli nel XIX secolo.

6. Del testo petitiano sopravvive una stampa (Petito I867), che funge da base sia per l'edizione in Tutto Petito, (1978: 307-328), sia per quella nell'antologia a cura di Franco Carmelo Greco (cfr. nota 5). Classico il riferimento al capitolo su Petito di Viviani (I992: 483-534).

7. Si rinvia a Cotticelli 2009: 455-510 e a Cotticelli (2013: 197-205). Sull'Ottocento, prescindendo dai contributi canonici sulla parodia (come Almansi; Fink 1976), si veda Sapienza (1998). 
Pellico. Petito non è nuovo all'incontro/scontro con un monumento testuale, e il suo graffio non è mai gratuitamente ridanciano, ma l'atteggiamento che assume di fronte a Francesca da Rimini va al di là dell'esegesi comica attenta ai dati salienti del plot. Come se volesse ridere non di Francesca, ma su Francesca. Sembra quasi che egli si allinei di istinto alle forti riserve di Foscolo, che ne aveva suggerito la distruzione tra le fiamme ${ }^{8}$, mostrandosi perplesso e irriverente di fronte a uno scenario suggestivo che agisce poco più che da contenitore a un esangue triangolo amoroso, svuotato delle sottili implicazioni dantesche, e via via caricato nelle pose stentoree, nelle frasi languide, nei moti impetuosi di Paolo, Lanciotto e della donna sotto lo sguardo pensoso di un padre nobile. Il tema è evanescente. Se la parodia è ricerca di altri sensi, qui non resta che ridere del teatro, delle sue forme e dei suoi mitologemi: attaccare quell'involucro di prassi e convenzioni che è la cifra più segreta e reale di una persistenza decennale nei cartelloni.

L'intento è quello di travisare uno "stravisamento", denunciando un'esilità che viene sistematicamente compensata dai virtuosismi di mostri sacri del palcoscenico, mentre potrebbe rivelarsi stucchevole e monocorde se appena cala la maestria espressiva e l'osservazione si fa più lucida e attenta. Di qui scaturisce il meccanismo della "commedia in commedia", attivato per di più da un inglese nel pubblico, chiaramente attratto dalla mitica risonanza di quello spettacolo, al "gran chiasso di voci confuse, rumore di robba caduta» e al colpo di pistola che interrompe la sinfonia da poco avviata. Tocca a Pulcinella spiegare l'accaduto:

Signori miei, dovete sapere che questa sera quelli che doveano rappresentare la tragedia era na compagnia de provincia che, truvannese de passaggio pe chesta bella metropoli, ha cercato in piacere a o 'mpressario de fa' na serata de beneficio, e siccome le mancava la primma donna, trovandosi di residenza in Napoli la signora donna Giulietta Pappabene, che dovea spusa' o marchesino Spiennesempre, questa se prestava gratuitamente, come avete potuto vede' d'o manifesto. S'è data la combinazione che 'ncoppe a li scene, per ordine del direttore, o marchesino non ha potuto sagli', a signora s'è pigliato collera, o marchesino s'è corrivato e pe dispietto s'è iuto a mettere dinto a nu palco de terza fila, addo' nce stevene certe ballarine, anche conoscitrice del marchesino. Nfraditanto steveme facenne l'opera: la prima donna, da dinto a no pertuso d'o telone, che per lo più e pertuse sono i segretarii dei gabinetti amorosi, ha avvistato o marchesino dinto o palco de le ballarine... Signori miei avisseve avuta sta' 'ncoppa a li scene, pe vede' che è succieso. A la primma donna le so' venute quattro convulsioni, una dinto a n'autra, o primmo amoroso la ristorava nel camerino, che per lo più questi sono dritti e spuzzuliature devolute ai primi amorosi: o portacesta de la primma donna, pe s'abbusca' no rialo è iuto a avvisa' a lu marchesino dinto o palco, ca la signora fa ammore cu lu prim-

8. Per questo commento cfr. Pellico (1985: 262). 
mo amoroso... È sciso o marchesino a dinto o palco, ha dato na vuttata a don Giuvanne, è trasuto dinto, ha truvato a chillo dinto o cammerino e ha ditto: "Ah! ingrata traditrice spetrice, così tratti un infelice che per te s'è ridotto n'alice senza camice". Poi, autannese 'n faccia a o primmo amoroso, ha ditto: "Signore, di quest'affronto ne voglio un offesa domani ai Ponti rossi, all'alba: duello sino all'ultimo sanguinaccio, senza patrino pecchè i patri nostri non sappiamo quanti sono". O primmo amoroso, che faceva Paolo, ha vutata a sciabola da parte do cuozzo e ha sciaccato o marchesino. O marchesino ha cacciato o rivolte e ha menata na pistolettata, che l'ha cuoveta 'n faccia a lu cappiello do patre nobile e nce l'ha sfunnato. La caratterista, pe la paura, è iuta pe fui' e è sciuliata ienne co promontorio 'n terra e non so quale parte si ha lesionata. La mugliera do custode do triato, ch'era gravida de nove mise, sentenne a botta steva dinto o cammerine do luminario: era grossa prena, era uscita e conti ed è figliate, e non se sape si è masculo o è femmena e se sta aspettanno la vammana per una tale verifica. O capo comico, vedenno stu 'mbruoglio, s'ha arrunzato li vestiti, s'ha arrunzato l'introito e se ne è fuiuto cu l'autro riesto de la compagnia. $\mathrm{O}$ marchesino e l'amoroso so' stati arrestati, la primma donna se l'ha portata o chirurgo a la casa, tutto o riesto de gente che stevene 'ncoppa e scene se ne so' fuiute e so' rummasto io sulo e... (Greco I995: I30-I3I)

C'è del genio in questo accumulo di disavventure sciorinato senza tregua davanti agli spettatori: innanzitutto, l'accenno alla compagnia di provincia, alla serata di beneficio e all'approssimazione dell'allestimento, con un'allusione neppure tanto velata alla Sadowsky e alla sua ascesa sociale per via di matrimonio (lei che per Napoli è "una sorta di monumento perenne al personaggio ed alla tragedia di Francesca del Pellico") (Greco 1995: 137); quindi, un lessico specialistico dispiegato a designare le persone coinvolte in questa vicenda (prima donna, primo amoroso, porta cesta, caratterista, capocomico), con enfasi su usi e costumi dietro e fuori le quinte (ballerine che accolgono il marchesino; primi amorosi che confortano colleghe in crisi...), fino alla fuga del capocomico con le suppellettili e il "bottino" della serata; le inserzioni di discorso diretto, dove l'intonazione greve e sostenuta contraddice la levità dell'effetto comico. È forse questo lo sberleffo più intelligente: prima dell'apertura di sipario si è già consumata una Francesca da Rimini, l'esplosione di un dramma della gelosia sorretto da equivoci e sospetti, e la finzione di un racconto ha il sopravvento sulla finzione prevista per la serata, per altro non dissimile nella sostanza a quel che al pubblico è lasciato immaginare.

La deflagrazione della parodia in questo gioco metateatrale è funzionale a mettere in discussione il valore di una storia che può ridursi alle tensioni di un adulterio e che solo una certa scaltrezza compositiva (gli equilibri che offre ai ruoli più rilevanti e dunque la possibilità di garantire eguali opportunità a un gruppo di interpreti di rango) e il suo radicamento in un immaginario letterario prestigioso - accanto a uno specifico battage pubblicitario in diverse occasioni - hanno consentito di resistere nel tempo. Quel che poi Petito offre 
di seguito è per l'appunto una sintesi estrema dell'azione originaria: l'incontro fra Guido e Lanciotto, l'apparizione di Francesca (per di più con Pulcinella en travesti), l'arrivo di Paolo, la confessione dei due cognati e il finale luttuoso. Quasi un prosciugamento, forse esaltato dall'accortezza con cui si lavora al travisamento degli altri codici, in primis i costumi. Alle fogge eleganti e ricercate di cinquant’anni prima («Francesca è vestita di raso bianco dalla cintura in giù e le maniche. Il busto e le scarpe sono di color cenerino scuro. Paolo ha il vestito sino alla cintura di velluto cremisino, i calzoni di pelle gialli. Il berretto è cremisino e le piume sono parte bianche e parte turchino chiaro. Lanciotto è vestito tutto di color verde scuro, eccettuando gli accessori che sono gialli»" ${ }^{9}$ si contrappone il richiamo derisorio delle istruzioni di scena "napoletane":

FranCESCa Vestita con una lunga camicia da notte, una cuffia idem in testa e un lenzuolo appeso dietro le spalle, come manto.

Guido Calzato di corto, con abito alla spagnola di sopra, e manto alla romana; sciaccò da militare: parrucca nera e barba bianca.

LANCIOTTO Scemisa alla romana; un grosso brando: collana formata di una funicella con riverbero di lame appeso: mostaccio bianco e mosca nera.

PAOLO bacile da barbiere in testa, da servire per elmo; grossa spadancia e lancia: un soprabito alla militare di sopra, con corazza alla romana: una grossa cartiera a tracolla: parrucca nera e baffi rossi.

PAGGIo Da cocchiere d'affitto. (Greco I995: I28)

Solo il breve incipit è identico, "Vedermi dunque ella chiedea", vero e proprio senhal di una svolta nell'azione, ma subito l'abborracciata messinscena di Pulcinella e soci prosegue secondo i dettami della più consolidata tradizione dialettale, fra slittamenti di senso, fraintendimenti e - a ribadire lo squarcio della finzione entro la Francesca riveduta e "stravesata" - sottolineature dell'inadeguatezza del physique $d u$ rôle agli aristocratici esponenti della Romagna medioevale, non soltanto in un impatto visivo eloquente e atto a respingere ogni immedesimazione, ma entrando e uscendo dalla parte di tanto in tanto:

SCena II

Pulcinella, vestito con camicia da donna, lunga, e cuffia da notte, con un lenzuolo appeso dietro

Guido: (alzando le braccia per slanciarsi)

Francesca figlia.

9. È la descrizione del disegno del quadro di casa Alario di cui parla Pellico in una lettera al fratello Luigi dell'8 marzo I8I8, per la quale cfr. Pellico (I98I: 264). 
Francesca: O Guido padre.

Signore: (dal palco) Ma signori miei, questa mi sembra un'indecenza!

Come, una donna si presenta al pubblico in camicia!

Pulcinella: (slanciandosi dal padre e facendosi avanti) Mio signo', ho l'onore di dirve che lei sbaglia, perché io non son donna, e poi ho creduto di far bene vestennome accussì, perché la storia dice che Francesca era molto calorosa.

INGLESE: Oh jèse, seguitate, piaciuto calorosa.

Guido: (dopo di queste parole, corre a Francesca, l'afferra e stringendosela al seno dice)

Che io ti stringa, o zuccarin d'amore.

Pulcinella: Gue' non stregnere tanto, viecchio veziuso.

Guido: (seguitando, senza badare alle parole di Pulcinella)

Lungi da te

Di lagrime versai quasi un barile. (Greco I995: I3I-I32)

senza tralasciare anche l'esibizione dei segreti del mestiere:

LANCiOtTo: Sarebbe vero?

Primo Violino: (dalla platea) Ne', faciteme nu piacere don Asdru', pecché o scudiere ieva vestuto a cucchiere?

Asdrubale: (un poco imbrogliato) Ecco qua...perché fra scudiero e cocchiere nce è una certa relazione, ecco pecché chillo, non putennose vesti' a scudiere, pe nun tradi' o carattere, s'è vestuto da cucchiere.

INGLESE: Jèse, cocchiere e scocchiere essere tutto una cosa.

Suggeritore: Ne', don Asdru', vuie volite seguita', ca nce sta a parola de filo, si no nun pozzo attacca' a bussata de Paolo.

Asdrubale: Se', iammoncenne trenta carri'. Sarebbe vero, Paolo qui! Oh, contento! (Greco 1995: I32-I33)

Altrove si sollecita la memoria degli astanti attraverso l'affettazione di una sintassi recitativa "italiana":

Lanciotto: Purtroppo ella ha bisogno di decotto.

Guido: E qual ne è la causa?

Lanciotto: (grattandosi la pancia) Chi lo sa.

Pulcinella: (con posa alla Ristori) Tu il sei! (Greco 1995: 132)

mentre il finale foscoliano di Pellico ("Guido Ferma / già è tuo quel sangue; e basta, onde tra poco / inorridisca al suo ritorno il sole"; Pellico I98I: 308; atto $\mathrm{V}$, scena ultima) cede il passo a un "Quadro finale come a concerto" preceduto dall'ultima, irriverente $g a g$, con una clausola tragica posta a commento di uno sgraziato "incidente":

(Guido, che teneva le falde della giamberga del suggeritore strette fra le mani, nel cadere che fa, quelle gli rimangono in mano, scucendosi dalla giamberga, e lasciando vedere un calzone tutto rattoppato) 


\section{GuIDo: (inorridito dice)}

Quale vista crudel s'offre a' miei sguardi! (Greco I995: 136)

La rinuncia a ogni verosimiglianza - accentuata anche dall'alternarsi di Pulcinella/Francesca nelle indicazioni di testo - risulta proporzionale al piacere del nonsense, o della trasformazione in senso quotidiano e ordinario dei passaggi magniloquenti. Basterebbe por mente al colloquio fra Paolo e Francesca, dove è l'uomo a farsi testimone dell'antica fiamma, ma Pellico intarsia le reminiscenze dantesche in un discorso paratattico, franto, atto a richiamare a un tempo l'attenzione alla parola (la Commedia è il libro incriminato) e a un gesto impossibile nel codice recitativo ottocentesco ("Questi, che mai da me non fia diviso / La bocca mi baciò tutto tremante"; If. V, I36-I37)), mentre Petito dissacra la situazione proprio attraverso l'ostensione ridicola dell'oggetto libro, veicolo della "tentazione":

\section{Pellico}

PaOlo: E presso al lago

in mezzo ai fior prosteso, io sospirando le tue stanze guardava; e al venir tuo tremando sorsi. Sopra un libro attenti non mi vedeano gli occhi tuoi; sul libro ti cadeva una lagrima... Commosso mi t’accostai. Perplessi eran miei detti, perplessi pure erano i tuoi. Quel libro mi porgesti e leggemmo. Insiem leggemmo "di Lancillotto come amor lo strinse. Soli eravamo e senza alcun sospetto..." Gli sguardi nostri s'incontraro...il viso mio scolorossi...tu tremavi...e ratta ti dileguasti.

(Pellico 198I: 29I; atto III, scena seconda)

\section{Petito}

Francesca: Per carità, non dir più ciuccerie, Pensa un istante alle faccende mie.

PaOlo: (con rimprovero dolce, brusco)

Io t'ebbi sempre dentro al cor scolpita:

Tu invece, ingrata, hai la mia fe' tradita.

Mira. (e presenta il libraccio che porta ligato a tracollo)

FRANCESCA: (con finto riso)

Che veggo, che libraccio è questo.

PaOlo: Più nol ravvisi, stupefatto io resto.

È il pappucon, che leggevamo un giorno.

Quando fummo in Acerra a far soggiorno

Soli eravamo, e sotto ombrosa frasca, 
Mangiando il casatel cu ll'ove Pasca,

Li lacreme d'o monte eran cascate

Su tre o quattro delle sue facciate:

Vedi, <che> ancor ne gronda: no vide

Che fet'e vino.

(Greco I995: I33)

Il valore icastico attribuito dallo sgangherato interprete di Paolo a un copione travolto dalle faccende conviviali della troupe è il suggello a un'operazione di demistificazione che punto al riso non senza prendere le distanze da un teatro di parola connaturato allo stile grandattoriale. Petito è uomo di scena, non di libro ${ }^{\mathrm{IO}}$, e anzi fatica moltissimo a trasformarsi in autore del proprio testo. Il suo è un caso emblematico di scrittura adeguata ai ritmi e alle movenze dello spettacolo e dell'oralità, con collaborazioni che inquinano ulteriormente un processo complicato di passaggio dall'appunto e dalla voce alla stampa ${ }^{\text {II }}$. Eppure, lontano da proclami, polemiche e intellettualismi, egli avverte il disagio di un repertorio inattuale, e inattuale perché distante. La complicità con il pubblico che scatta nella parodia - e che appare tra le strategie della contraffazione - punta forse l'indice contro la sopraffazione del pubblico che quel titolo classico reca indissolubilmente con sé. Qui Petito pare riprendere la vis morale di Dante e la sua denuncia del binomio lussuria/letteratura cortese, sferrando il suo attacco - bonario ma incisivo - al teatro "serioso", non "serio": quello che non è né intrattenimento felice (e creazione di un circuito comunicativo), né autentico dibattimento. Solo inesausta celebrazione di sé. Una lezione circoscritta, che le periodiche ri-scoperte della tradizione dialettale da quel lontano 1866 dimostrano di non avere mai opportunamente dimenticato.

IO. Il riferimento è alla diade teorizzata da Taviani (I995).

II. Sulla questione cfr. Petito (I989: 9-28) e pp. 8I-85 e Petito (I990: 473-502). 

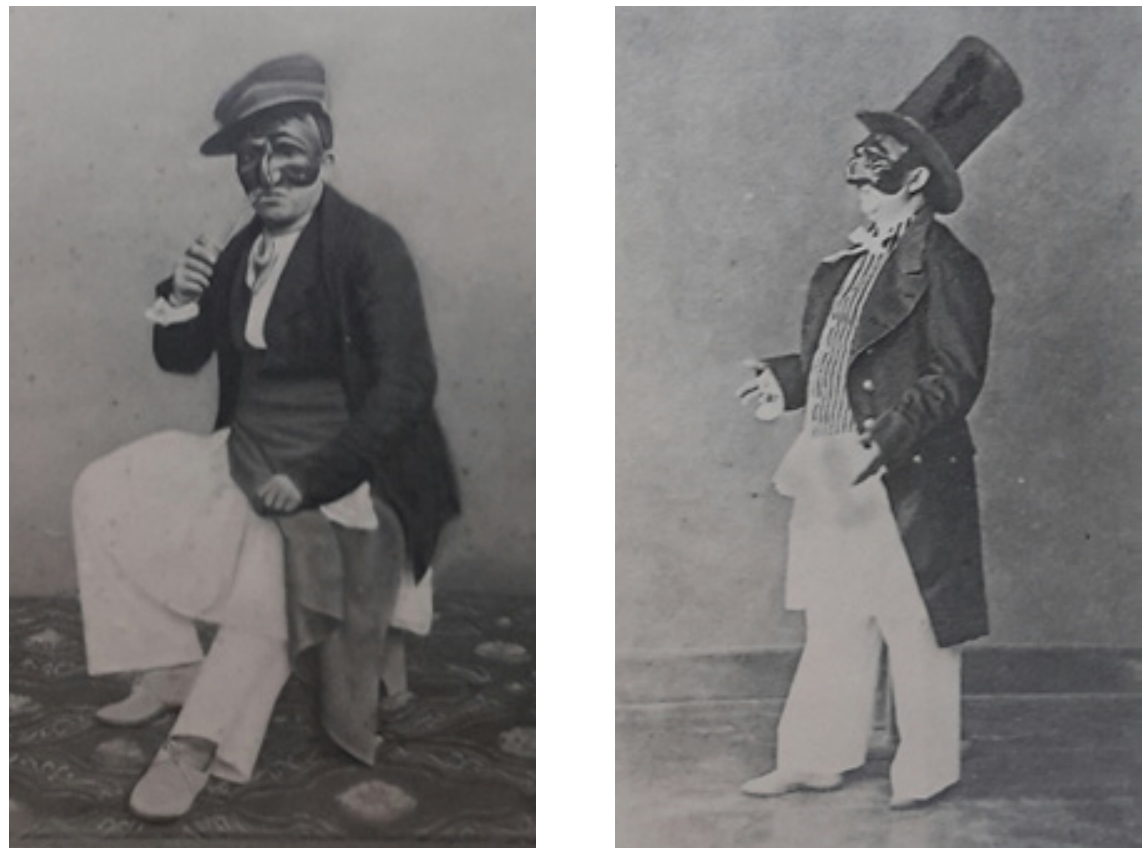

Figg. I e 2: Antonio Petito.
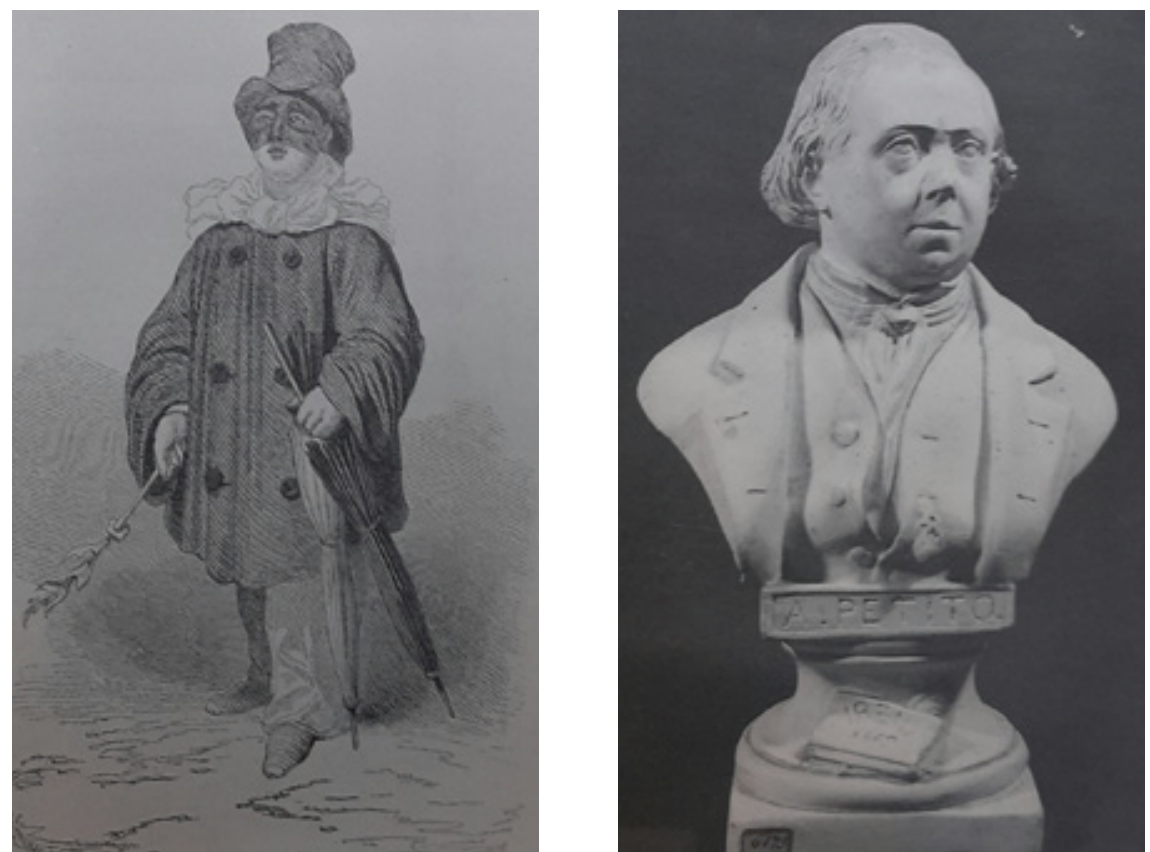

Fig. 3: Antonio Petito come Pulcinella; fig. 4: busto di Antonio Petito. 


\section{BibliografiA}

Almansi, G., Fink, G., 1976, Quasi come. Parodia come letteratura, letteratura come parodia, Milano, Bompiani.

Borrelli, C., 1995 Francesca da Rimini nella fruizione ottocentesca mediata da Francesco Saverio Salfi, in Matteo Palumbo e Vincenzo Placella (a cura di), Miscellanea di studi in onore di Raffaele Sirri, Napoli, Federico e Ardia, pp. 47-62.

Cotticelli, F., 2009, "Il teatro recitato", in Storia della musica e dello spettacolo a Napoli. Il Settecento, a cura di Francesco Cotticelli e Paologiovanni Maione, Napoli, Turchini edizioni, pp. 455-510

—, 20I3, "Sulle caratteristiche "nazionali" nel teatro napoletano dagli anni Settanta in relazione alle compagnie forestiere", in L'idea di nazione nel Settecento, a cura di Beatrice Alfonzetti e Marina Formica, Roma, Edizioni di Storia e Letteratura, pp. 197-205.

Farina, F., 2006, Francesca da Rimini. Sulle tracce di un mito, Romagna Arte e Storia, 78 , pp. 5-I34.

Taviani, F., I995, Uomini di scena, uomini di libro. Introduzione alla letteratura teatrale italiana del Novecento, Bologna, Il Mulino, I995.

Greco, G., 1995, La scena illustrata. Teatro, pittura e città a Napoli nell'Ottocento, Napoli, Tullio Pironti.

Pellico, S., 198I, Francesca da Rimini, in Il teatro italiano. V. La tragedia dell'Ottocento, tomo primo, a cura di Emilio Faccioli, Torino, Einaudi, pp. 259-308.

Petito, A., I867, Francesca da Rimini, tragedia a vapore stravesata da Pulicenella cetrulo, da D. Asdrubale Barilotti, da Monzù Patrecutenella e da Schiattamuorton. Bizzarria comica scritta dal Signor Antonio Petito, Napoli, Stabilimento Tipografico de' fratelli De Angelis.

—, 1978, Tutto Petito, a cura di Ettore Massarese, Napoli, Luca Torre editore, II.

-, I989, 'A palomela, a cura di Franco Carmelo Greco, con Palummella zompa e vola di Tato Russo, Napoli, Bellini editrice, pp. 9-28 e pp. 8I-85

—, I990, "Petito-Pulcinella: una maschera della storia", in Pulcinella. Una maschera tra gli specchi, a cura di Franco Carmelo Greco, Napoli, E.S.I., I990, pp. 473-502.

Sapienza, A., 1998, La parodia dell'opera lirica a Napoli nell'Ottocento, Napoli, Guida.

—, 2003, "Parodia e metateatro. Francesca da Rimini di Antonio Petito", Misure critiche, nuova serie, II, I-2, pp. 45-59

Seller, F., 1989, "Fanny Sadowsky", in Il teatro Mercadante. La storia, il restauro, a cura di Tobia Raffaele Toscano, Napoli, Electa, pp. 197-200.

Viviani, V., 1992 [1969], Storia del teatro napoletano, Napoli, Guida, pp. 483-534.

Si può vedere qui http://www.vigata.org/attivita/rimini.shtml una messinscena moderna della farsa Francesca da Rimini. Tragedia a vapore, di Antonio Petito. Interpreti: Carlo Giuffrè (Don Anselmo), Aldo Giuffrè (Don Gennaro), Mimmo Brescia (Minicuccio), Clara Bindi (Signora Francesca), Pino Salis (Suggeritore). Regia Teatrale di Aldo e Carlo Giuffrè. Luci di Carlo Natali. Regia Televisiva di Andrea Camilleri. È andata in onda il 20 gennaio I980 sul Secondo Canale della Rai [NdR].

Le immagini, che riproducono alcuni momenti della vita dell'autore-attore napoletano, sono tratte da Greco (1995) [NdR]. 
